\title{
Tachyon Monopole
}

\author{
Xin-zhou L: and Dao-jun Liu \\ Shanghai United Center for Astrophysics(SUCA), \\ Shanghai Normal University, 100 Guilin Road, Shanghai 200234, China
}

(Dated: June 18, 2021)

\begin{abstract}
The property and gravitational field of global monopole of tachyon are investigated in a four dimensional static space-time. We give an exact solution of the tachyon field in the flat space-time background. Using the linearized approximation of gravity, we get the approximate solution of the metric. We also solve analytically the coupled Einstein and tachyon field equations which is beyond the linearized approximation to determine the gravitational properties of the monopole solution. We find that the metric of tachyon monopole represents an asymptotically AdS space-time with a small effective mass at the origin. We show that this relatively tiny mass is actually negative, as it is in the case of ordinary scalar field.
\end{abstract}

PACS numbers: 11.27.+d, 98.80.Cq, 11.25.-w

\section{Introduction}

Recently, pioneered by Sen [1], the study of non-BPS objects such as non-BPS branes, brane-antibrane configurations or space-like branes [2] has been attracting physical interests in string theory. Sen showed that classical decay of unstable D-brane in string theories produces pressureless gas with non-zero energy density. The basic idea is that the usual open string vacuum is unstable but there exists a stable vacuum with zero energy density. There is evidence that this state is associated with the condensation of electric flux tubes of closed string [3]. These flux tubes described successfully using an effective Born-Infeld action [4]. The tachyon rolling towards its minimum at infinity as a dark matter candidate was also proposed by Sen [3]. Sen have also studied the Dirac-Born-Infeld Action on the Tachyon Kink and Vortex [6]. Gibbons took into account coupling to gravitational field by adding an Einstein-Hilbert term to the effective action of the tachyon on a brane, and initiated a study of "tachyon cosmology" [5]. Several authors have investigated the process of rolling of the tachyon in the cosmological background [7, [8].

Various topological defects such as domain wall, string and monopole could be formed by the symmetry-breaking phase transitions in the early universe and their existence has important implications in cosmology 9 , 10]. The symmetry breaking model of ordinary scalar field can be prototypically written as

$$
L=\frac{1}{2} \partial_{\mu} \phi^{a} \partial^{\mu} \phi^{a}-V(f)
$$

where $\phi_{a}$ is a set of scalar fields, $a=1, \ldots, N, f=\left(\phi^{a} \phi^{a}\right)^{\frac{1}{2}}$. The model has $\mathrm{O}(\mathrm{N})$ symmetry and admits domain wall, string and monopole solutions for $N=1,2$ and 3, respectively [11]. Usually, the potential $V(f)$ has a minimum at a finite non-zero value of $f$. On the other hand, in Ref. [12, 13], Cho and Vilenkin investigated the defects in models where $V(f)$ has a local maximum at $f=0$ but no minima; instead, it monotonically decrease to zero at $f \rightarrow \infty$. And they called this kind of defects "vacuumless defects".

Global monopole, which has a global $\mathrm{O}(3)$ symmetry spontaneously broken to $\mathrm{U}(1)$, is one of the most interesting above mentioned defects. The property of the global monopole in curved space-time, or equivalently, its gravitational effects, was firstly studied by Barriola and Vilenkin 14]. When one considers the gravity, the linearly divergent mass of global monopole has an effect analogous to that of a deficit solid angle plus that of a tiny mass at the origin. Harari and Loustò [15], and Shi and Li [16] have shown that this small gravitational potential is actually repulsive. A new class of cold stars, addressed as D-stars(defect stars) have been proposed by Li et.al. [17, 18]. One of the most important features of such stars, comparing to Q-stars, is that the theory has monopole solutions when the matter field is absent, which makes the D-stars behave very differently from the Q-stars. Furthermore, if the tachyon field $T$ rolls down from the maximum of its potential and the universe does not inflate, the quantum fluctuations produced various topological defects during spontaneous symmetry breaking. It is therefore of importance to investigate the property and the gravity of the topological defects of tachyon, such as vortex [19], kink [20] and monopole, in the

*Electronic address: kychz@shnu.edu.cn 
static space-time. In the next section, equations of tachyon monopole are presented. In sect. 3, we find an analytical solution of tachyon monopole in the flat space-time background and discuss its property. We show that because the tachyon field rolls down to its ground without oscillating about it, the tachyon monopole becomes very diffuse, just as the "vacuumless monopole" studied in Ref. [12]. In sect. 4, the gravitational field of tachyon monopole are investigated by using linearized gravity approximation and considering the coupled Einstein and tachyon field equation which is beyond linearized approximation, respectively. We will find that the metric of tachyon monopole represents an asymptotically AdS space-time with a small effective mass at the origin which is actually negative. If tachyon monopole existed, they would share with other topological defects, such as domain walls and strings, curious and rather unconventional gravitational effect. Finally, we give some brief discussions.

\section{Equations of motion}

A general static, spherically-symmetric metric can be represented as

$$
d s^{2}=B(r) d t^{2}-A(r) d r^{2}-r^{2} d \Omega^{2}
$$

where $d \Omega^{2}$ is the metric on a unit 2-sphere. And the Lagrangian density of rolling tachyon with potential $V(\phi)$, which couples to the Einstein gravity, can be written as the following Born-Infeld form:

$$
\begin{aligned}
L & =L_{R}+L_{T} \\
& =\sqrt{-g}\left[\frac{R}{2 \kappa}-V(|T|) \sqrt{1-g^{\mu \nu} \partial_{\mu} T^{a} \partial_{\nu} T^{a}}\right]
\end{aligned}
$$

where $T^{a}$ is a triplet of tachyon fields, $a=1,2,3$ and $g_{\mu \nu}$ is the metric. The field configuration describing a monopole is given by

$$
T^{a}=f(r) \frac{x^{a}}{r}
$$

where $x^{a} x^{a}=r^{2}$. From the Lagrangian density (3), metric (2) and the above configuration of the field(41), we can obtain the following Euler-Lagrange equation:

$$
\frac{1}{V} \frac{d V}{d f}+\frac{2 f}{r^{2}}=\frac{1}{A}\left[f^{\prime \prime}+\left(\frac{B^{\prime}}{2 B}-\frac{A^{\prime}}{2 A}+\frac{2}{r}\right) f^{\prime}-f^{\prime} \frac{\frac{f^{\prime} f^{\prime \prime}}{A}-\frac{f^{\prime 2} A^{\prime}}{2 A^{2}}+\frac{2 f}{r^{2}}\left(f^{\prime}-\frac{f}{r}\right)}{1+\frac{f^{\prime 2}}{A}+\frac{2 f^{2}}{r^{2}}}\right]
$$

where the prime denotes the derivative with respect to $r$. The Einstein equations read:

$$
\begin{aligned}
& \frac{1}{r^{2}}-\frac{1}{A}\left(\frac{1}{r^{2}}+\frac{1}{r} \frac{A^{\prime}}{A}\right)=\kappa T_{0}^{0} \\
& \frac{1}{r^{2}}-\frac{1}{A}\left(\frac{1}{r^{2}}+\frac{1}{r} \frac{B^{\prime}}{B}\right)=\kappa T_{1}^{1}
\end{aligned}
$$

where the energy-momentum tensor $T_{\nu}^{\mu}$ of the system are given by

$$
\begin{gathered}
T_{0}^{0}=V(f) \sqrt{1+\frac{f^{\prime 2}}{A}+\frac{2 f^{2}}{r^{2}}} \\
T_{1}^{1}=\frac{V(f)\left(1+\frac{2 f^{2}}{r^{2}}\right)}{\sqrt{1+\frac{f^{\prime 2}}{A}+\frac{2 f^{2}}{r^{2}}}}
\end{gathered}
$$




$$
T_{2}^{2}=T_{3}^{3}=\frac{V(f)\left(1+\frac{f^{\prime 2}}{A}+\frac{f^{2}}{r^{2}}\right)}{\sqrt{1+\frac{f^{\prime 2}}{A}+\frac{2 f^{2}}{r^{2}}}}
$$

and the rest are zero. Obviously, the configuration of the system depends on the tachyon potential $V(T)$. However, the explicit form of the potential is not clear now. According to Sen [3] , the potential should have an unstable maximum at $T=0$ and decay exponentially to zero when $T \rightarrow \infty$. There are lots of functional forms that satisfy the above two requirements. In the following sections, we choose the tachyon potential as follows:

$$
V(f)=M^{4}\left(1+3 \lambda f^{4}\right)^{1 / 6} \exp \left(-\lambda f^{4}\right)
$$

where $M$ and $\lambda$ are two constants and both are greater than zero. Obviously, this potential dacays faster than the exponential one for large $f$, but qualitatively, it has the similar property as the exponential potential.

\section{The analytical solution of tachyon monopole in flat space-time background}

In flat space-time background, the Euler-Lagrange equation (15) can be reduced to the following equation:

$$
\frac{1}{V} \frac{d V}{d f}+\frac{2 f}{r^{2}}=f^{\prime \prime}+\frac{2 f^{\prime}}{r}-f^{\prime} \frac{f^{\prime} f^{\prime \prime}+\frac{2 f}{r^{2}}\left(f^{\prime}-\frac{f}{r}\right)}{1+f^{2}+\frac{2 f^{2}}{r^{2}}}
$$

and the energy density of the system is written as

$$
T_{0}^{0}=V(f) \sqrt{1+f^{\prime 2}+\frac{2 f^{2}}{r^{2}}} .
$$

For the potential (11), the equation (12) has a simple exact solution

$$
f(r)=\lambda^{-\frac{1}{4}}\left(\frac{r}{\delta}\right)^{-1}
$$

where $\delta=\lambda^{-1 / 4}$ is the size of the monopole core, and the corresponding energy density (13) can be rewritten as

$$
T_{0}^{0}=M^{4}\left[1+3\left(\frac{\delta}{r}\right)^{4}\right]^{2 / 3} \exp \left[-\left(\frac{\delta}{r}\right)^{4}\right] .
$$

The total energy of the tachyon monopole is

$$
\mu(R)=4 \pi \int_{0}^{R} T_{0}^{0} r^{2} d r \sim 4 \pi M^{4}\left(\frac{R^{3}}{3}-\frac{1}{\lambda R}\right),
$$

where the cutoff radius $R$ is set by the distance to the nearest anti-monopole. We find easily from Eq. (16) that tachyon monopoles are very diffuse objects with most of the energy distributed at large distances from the monopole core, and we expect their space-time to be substantially distinct from the ordinary case. They are much more diffuse than ordinary global monopoles which have $\mu(R) \propto R$, so that the gravitational effect of this configuration is equivalent to that of a deficit solid angle in the metric with a negative mass at the origin. Furthermore, they are similar to the vacuumless monopoles which have $\mu(R) \propto R(R / \delta)^{4 /(n+2)}$, so that most of the energy is also diffused at large distance 12]. By analogy with vacuumless monopoles and ordinary monopoles, the typical distance between the monopoles at time $t$ is $R \sim t$ [12], apart from the close monopole-antimonopole pairs which are about to annihilate. Like ordinary monopoles, one of the cosmological bound to tachyon monopole is that it should not overclose the universe. This 
means that the energy density in monopoles $\rho_{m}$ today should be less than the critical density of the present universe. The ratio of monopole energy density to critical density $\Omega_{m}$ at time $t$ is

$$
\Omega_{m}(t)=\frac{\rho_{m}}{\rho_{c}(t)} \sim \frac{32 \pi M^{4} t^{2}}{3 M_{p}^{2}}
$$

where $M_{p}$ is the Planck mass. Therefore, the parameter $M$ should satisfies the condition $M \lesssim 10^{-3} \mathrm{eV}$ in order to avoid conflicting present cosmological observations.

\section{The gravitational field of tachyon monopole}

Firstly, the space-time of tachyon monopole will be studied using the linearized gravity approximation in order to show the main features of its metric in a simple manner. And then, we will solve analytically the coupled Einstein and tachyon field equations which is beyond the linearized approximation to rigorously determine the gravitational properties of the monopole solution.

\subsection{Linearized approximation}

In this subsection, we study the space-time of global tachyon monopole using the linearized gravity approximation. We shall first consider the Newtonian approximation. The Newtonian potential $\Phi$ can be found for the equation

$$
\nabla^{2} \Phi=\frac{\kappa}{2}\left(T_{0}^{0}-T_{i}^{i}\right)
$$

For the tachyon monopole, $f(r)$ is given by Eq. (14) and

$$
T_{0}^{0}-T_{i}^{i} \simeq-2 M^{4}
$$

at $r>>\delta$. Then the solution of Eq. (18) is

$$
\Phi(r) \simeq-\frac{4 \pi M^{4}}{3 \lambda M_{p}^{2} f^{2}}
$$

The linearized approximation applies as long as $|\Phi(r)|<<1$, which is equivalent to $f>>\sqrt{\frac{4 \pi}{3 \lambda}} \frac{M^{2}}{M_{p}}$. Therefore, we should require the parameters $\lambda$ and $M$ satisfy

$$
\lambda^{-1 / 4}<<R<<\sqrt{\frac{3}{4 \pi}} \frac{M_{p}}{M^{2}}
$$

where $R$ is the distance to the nearest anti-monopole. Next, we express the metric functions $A(r)$ and $B(r)$ as

$$
A(r)=1+\alpha(r), \quad B(r)=1+\beta(r) .
$$

Linearizing in $\alpha(r)$ and $\beta(r)$, and using the flat space expression (14) for $f(r)$, the Einstein equations (6) and (7) can be written as follows

$$
\frac{\alpha^{\prime}}{r}+\frac{\beta^{\prime}}{r}=\kappa M^{4}\left(\frac{\delta}{r}\right)^{4}\left[1+3\left(\frac{\delta}{r}\right)^{4}\right]^{-1 / 3} \exp \left[-\left(\frac{\delta}{r}\right)^{4}\right]
$$

and

$$
\beta^{\prime \prime}+\frac{2 \beta^{\prime}}{r}=-\kappa M^{4}\left[2+3\left(\frac{\delta}{r}\right)^{4}\right]\left[1+3\left(\frac{\delta}{r}\right)^{4}\right]^{-1 / 3} \exp \left[-\left(\frac{\delta}{r}\right)^{4}\right]
$$


The solution of external metric is easily found

$$
d s^{2}=\left(1-\frac{\kappa M^{4}}{3} r^{2}\right) d t^{2}-\left(1+\frac{\kappa M^{4}}{3} r^{2}-\frac{\kappa M^{4}}{2 \lambda r^{2}}\right) d r^{2}-r^{2} d \Omega^{2} .
$$

The metric (25) can be expressed by the form of Newtonian potential

$$
d s^{2}=(1+2 \Phi) d t^{2}-\left[1-2 \Phi\left(1-\frac{3}{2}\left(\frac{\delta}{r}\right)^{4}\right)\right] d r^{2}-r^{2} d \Omega^{2} .
$$

It is obvious that the metric (25) is linearized approximation of an AdS space-time.

\subsection{Coupled Einstein and tachyon field equations}

In the above subsection, the linearized approximation of gravity is used, which is available as long as the Newtonian potential is much smaller than unit. In fact, for the spherical symmetric metric (2), there is a general solution of the Einstein equations with spherically symmetric energy-momentum tensor $T_{\nu}^{\mu}$ which takes the form as [18, 21]

$$
A(r)^{-1}=1-\frac{\kappa}{r} \int_{0}^{r} T_{0}^{0} r^{2} d r
$$

and

$$
B(r)=A(r)^{-1} \exp \left[\kappa \int_{\infty}^{r}\left(T_{0}^{0}-T_{1}^{1}\right) A(r) r d r\right]
$$

Substututjion of Eq.(8) on the Eq.(27) yields

$$
A^{-1}=1-\frac{\kappa M^{4}}{r} \int_{0}^{r}\left(1+3 \lambda f^{4}\right)^{1 / 6} \exp \left(-\lambda f^{4}\right) \sqrt{1+\frac{f^{\prime 2}}{A}+\frac{2 f^{2}}{r^{2}}} r^{2} d r
$$

which is an integral equation for $A^{-1}$. Hence, we want to use the method of successive approximation which provides the solution as the limit of an infinite sequence of steps if these steps were carried out exactly, simply and almost identically each to the next so that the program is a relatively easy task. For the small $\kappa M^{4}$, we take $A^{-1}=1$ as the leading approximate value. Fortunately, in this case, we have an analytical solution of $f(r)$, Eq. (14). Therefore, by direct integrating Eq.(29), we have

$$
\begin{aligned}
A^{-1} & =1-\frac{\kappa M^{4}}{r} \int_{0}^{r}\left[1+3\left(\frac{\delta}{r}\right)^{4}\right]^{2 / 3} \exp \left[-\left(\frac{\delta}{r}\right)^{4}\right] r^{2} d r \\
& \simeq 1-\frac{\kappa M^{4}}{3} r^{2}+\frac{\kappa M^{4}}{r}\left[\delta^{3}+g(\delta)\right]-\frac{\kappa M^{4}}{\lambda} \frac{1}{r^{2}}
\end{aligned}
$$

for $r>\delta$, where

$$
g(\delta)=\int_{0}^{\delta}\left[1-\left[1+3\left(\frac{\delta}{r}\right)^{4}\right]^{2 / 3} \exp \left[-\left(\frac{\delta}{r}\right)^{4}\right]\right] r^{2} d r
$$

is a monotonously increasing and positive (but smaller than $\delta^{3}$ ) function. Analogously,

$$
\begin{aligned}
B & =A^{-1} \exp \left[\kappa M^{4} \int_{\infty}^{r}\left(\frac{\delta}{r}\right)^{4}\left[1+3\left(\frac{\delta}{r}\right)^{4}\right]^{-1 / 3} \exp \left[-\left(\frac{\delta}{r}\right)^{4}\right] r d r\right] \\
& \simeq 1-\frac{\kappa M^{4}}{3} r^{2}+\frac{\kappa M^{4}}{r}\left[\delta^{3}+g(\delta)\right]-\frac{3 \kappa M^{4}}{2 \lambda} \frac{1}{r^{2}}
\end{aligned}
$$


Eqs.(30) and (32) are the second approximate value of the metric which are beyond those obtained by linearized gravity approximation. It is easy to find that the gravitational field of tachyon monopole is strongly repulsive and the space-time becomes singular at a finite distance from the monopole core. From aforementioned analysis, we find that metric functions $A^{-1}(r)$ and $B(r)$ grow quadratically with the distance off its core, therefore the metric represents one of asymptotically AdS space-time. Furthermore, there is a relatively small gravitational potential of repulsive nature, corresponding to a mass $\sim M^{4}\left[\delta^{3}+g(\delta)\right]$ at the origin, but with opposite sign.

\section{Conclusions}

In this paper, we have studied the property and gravitational field of global monopole of tachyon matter in a four dimensional approximately spherically-symmetric space-time. We give an analytical solution of the tachyon field in the flat space-time background and in particular, using the linearized approximation of gravity and the method of successive approximation, we find the approximate solution of the metric, which denotes an AdS space-time with a negative mass at the origin. In contrast to the ordinary monopole, the tachyon monopoles are very diffuse objects with most of the energy distributed at large distances from the monopole core, and their space-time is substantially distinct from that of the ordinary monopole.

In previous papers 21], Li and Hao have investigated the ordinary global monopole in asymptotically dS/AdS spacetime and find that the mass of the monopole in the asymptotically dS space-time could be positive if the cosmological constant is greater than a critical value. This shows that the gravitational field of the ordinary global monopole could be attractive or repulsive depending on the value of the cosmological constant. We note the cardinal difference between the gravitational effects of tachyon and ordinary global monopole: for an ordinary global monopole, its main effect, due to its mass that grows linearly with the distance off its core, can be understood in terms of a deficit solid angle [14]; for a tachyon monopole, due to its metric functions $A^{-1}(r)$ and $B(r)$ that grow quadratically with the distance off its core, can be understood in terms of asymptotically AdS space-time. From our analysis of the coupled Einstein and tachyon field equations, we conclude that there is a relatively small gravitational potential of repulsive nature, corresponding to a negative mass at the origin. It is very interesting to study the motion of a test particle and light in the gravitational field of tachyon monopole. Although the test particle motion is quite different from the case of the Schwarzschild space-time with negative mass at the origin, the behaviors of light remain unchanged except a energy (frequency) shift. Some consequences of the existence of monopole with negative masses have been considered long ago [15, 16].In the general relativity, Bondi [22] showed that a system moves with constant proper acceleration, asymptotically approaching the speed of light. This runaway solution could be seen as a drawback for a theory that allows particle with negative mass. However, the tachyon monopoles are not isolated point particles, but extended sources, even though the effective negative mass reaches its asymptotic value very quickly outside the core. One cannot expect a global monopole by itself, but rather monopoles and anti-monopoles, which probably annihilated themselves very efficiently, so that the motion of a system with a monopole and a particle with equal positive mass will not runaway.

In the spirit of the string theory, the over all scale in the tachyon potential is $M^{4} \propto \frac{1}{(2 \pi)^{3} g_{s} l_{s}^{4}}$ and $\lambda^{1 / 4} \propto \frac{1}{l_{s}}$, where $g_{s}$ and $l_{s}$ are the coupling parameter and length scale of string, respectively. The bound on $\lambda$ and $M$ by Eq. (21) makes $g_{s}>>1$ which puts the filed theory description of string tachyons under doubt. Moreover, the bound also makes the tachyon very light that is an unacceptable situation from string theory point of view 23]. Therefore, it is not suitable for tachyon inspired from string theory to use linear approximation. However, if we adopt the point of view that the tachyon potential, Eq.(III) for example, comes purely from phenomenological context just as those considered in Ref. [24], there is no such problems.

Finally, it is worth noting that although we used the potential (11) in our discussion, all the conclusions will be preserved qualitatively if we use the potentials discussed by Sen [3], because these potentials have the similar quality as the potential.

\section{Acknowledgment}

This work is supported by National Natural Science Foundation of China under Grant No. 10473007 and Shanghai Municipal Education Commission (No.04DC28).

[1] A. Sen, JHEP 9806, 007(1998); A. Sen, JHEP 9808, 010(1998); A. Sen, JHEP 9808, 012 (1998).

[2] M. Gutperle and A. Strominger, JHEP 0204, 018 (2002) 
[3] A. Sen, JHEP 0204, 048 (2002); A. Sen, JHEP 0207, 065 (2002).

[4] A. Sen J. Math. Phys. 42, 2844(2001); G. W. Gibbons, K. Hori and P. Yi, Nucl. Phys. B596, 136 (2001).

[5] G. W. Gibbons, Phys. Lett. B537, 1 (2002).

[6] A. Sen, Phys.Rev. D68, 066008 (2003).

[7] X. Z. Li, J. G. Hao and D. J. Liu, Chin. Phys. Lett. 19, 1584 (2002); J. G. Hao and X. Z. Li, Phys. Rev. D66, 087301(2002); J. G. Hao and X. Z. Li, Phys. Rev. D68, 067501 (2003); D. J. Liu and X. Z. Li, Phys. Rev. D70 123504 (2004); J. G. Hao and X. Z. Li, Phys. Rev. D70 043529 (2004).

[8] X. Z. Li and X. H. Zhai, Phys. Rev.D67, 067501(2003); M. Sami, P. Chingangbam, T. Qureshi, Phys.Rev. D66, 043530(2002); A. Feinstein, Phys.Rev. D66, 063511(2002); D. Choudhury et al, Phys.Lett. B544, 231 (2002); S. Mukohyama, Phys. Rev. D66, 024009(2002); G. Shiu and I. Wasserman, Phys. Lett. B541, 6(2002); H. B. Benaoum, hep-th/0205140; T. Chiba, Phys. Rev. D66, 063514 (2002); J. M. Cline, H. Firouzjahi and P. Martineau, JHEP 0211, 041 (2002); S. Mukohyama, Phys. Rev. D66,123512 (2002); C. Kim, H. B. Kim, and Y. Kim, Phys. Lett. B552, 111 (2003); J. S. Bagla, H. K. Jassal and T. Padmanabhan, Phys. Rev. D67, 063504 (2003); T. Padmanabhan, Phys. Rept. 380235 (2003); C. Kim, H. B. Kim, Y. Kim and O. Kwon, JHEP 0303, 008 (2003); P. Singh, M. Sami, N. Dadhich, Phys. Rev. D68, $023522(2003)$.

[9] T. W. B. Kibble, J. Phys. A9, 1387(1976).

[10] A. Vilenkin, Phys. Rep. 121, 263(1985).

[11] C. M. Chen, H. B. Cheng, X. Z. Li and X. H. Zhai, Class. Quantum Grav. 13, 701(1996).

[12] I. Cho and A. Vilenkin, Phys. Rev.D59, 021701(1998).

[13] I. Cho and A. Vilenkin, Phys. Rev. D59, 063510(1999).

[14] M. Barriola and A. Vilenkin, Phys. Rev. Lett. 63, 341(1989).

[15] D. Harari and C. Loustò, Phys. Rev. D42, 2626(1990).

[16] X. Shi and X. Z. Li, Class. Quantum Grav. 8, 761(1991).

[17] X. Z. Li and X. H. Zhai, Phys. Lett. B364, 212(1995); J. M. Li and X. Z. Li, Chin. Phys. Lett.15, 3(1998); X. Z. Li, X. H. Zhai and G. Chen, Astropart. Phys. 13, 245(2000); X. Z. Li, H. B. Cheng and C. I. Kuo, Chin. Phys. Lett. 18, 4 (2001).

[18] X. Z. Li and J. Z. Lu, Phys. Rev. D62, 107501(2000).

[19] D. J. Liu and X. Z. Li, Chin. Phys. Lett. 20, 1678 (2003).

[20] C. Kim, Y. Kim and C. O. Lee, JHEP 0305, 020 (2003).

[21] X. Z. Li and J. G. Hao, Phys. Rev.D66, 107701(2002); J. G. Hao and X. Z. Li, Class. Quantum Grav. 20, 1703(2003).

[22] H. Bondi Rev. Mod. Phys. 29, 423(1957).

[23] M. Fairbairn, M. Tytgat, Phys. Lett. B546 1 (2002); M. Sami, P. Chingangbam, T. Qureshi, Pramana 62765 (2004).

[24] T. Padmanabhan, Phys. Rev. D66, 021301 (2002); M. Sami, Mod. Phys. Lett. A18, 691 (2003). 